\title{
PRDM14 mediates chemosensitivity and glycolysis in drug-resistant A549/cisplatin cells and their progenitor A549 human lung adenocarcinoma cells
}

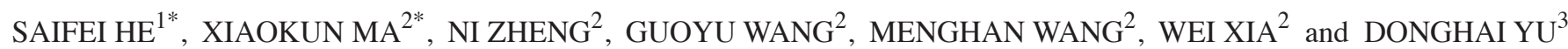 \\ Departments of ${ }^{1}$ Science and Research and ${ }^{2}$ Nuclear Medicine, The Seventh People's Hospital, Shanghai University of \\ Traditional Chinese Medicine, Shanghai 200137; ${ }^{3}$ Department of Science and Education, \\ The Municipal Health Commission, Shanghai 200125, P.R. China
}

Received July 13, 2020; Accepted November 20, 2020

DOI: $10.3892 / \mathrm{mmr} .2020 .11788$

\begin{abstract}
Recent studies have reported that aberrant PR domain zinc finger protein 14 (PRDM14) expression is associated with the therapeutic sensitivity of cancer cells to drugs. However, its role in lung adenocarcinoma (LUAD) remains unclear. The present study aimed to determine the functions of knockdown or overexpression of PRDM14 in the chemosensitivity and glycolysis of LUAD cells. PRDM14 expression was analyzed in lung cancer tissues from patients resistant and sensitive to cisplatin (DDP), as well as in LUAD cell lines A549 and DDP-resistant A549 (A549/DDP) using reverse transcription quantitative-PCR and western blotting. Additionally, apoptosis was analyzed by flow cytometry, and flow cytometry and biochemical analysis was used to analyze glycolysis, indicated by glucose uptake and lactate release. The results of the present study demonstrated that PRDM14 expression was upregulated in patients with DDP-resistant LUAD and DDP-resistant cell lines. Overexpression of PRDM14 suppressed the sensitivity of A549 cells to DDP and silencing of PRDM14 using shRNA targeting PRDM14 promoted the sensitivity of A549/DDP cells to DDP, compared with that in the respective control groups. In mice with xenograft tumors, knockdown of PRDM14 using shRNA targeting PRDM14 inhibited the A549/DDP cell-derived tumor growth
\end{abstract}

Correspondence to: Dr Donghai Yu, Department of Science and Education, The Municipal Health Commission, 990 Chengshan Road, Pudong New Area, Shanghai 200125, P.R. China

E-mail: yudonghai71@sina.com

Ms. Wei Xia, Department of Nuclear Medicine, The Seventh People's Hospital, Shanghai University of Traditional Chinese Medicine, 358 Datong Road, Pudong New Area, Shanghai 200137, P.R. China E-mail: awingxia_d@126.com

*Contributed equally

Key words: drug resistance, glycolysis, lung adenocarcinoma, PR domain zinc finger protein 14 compared with scramble shRNA. The results of the glycolysis assays demonstrated that PRDM14 silencing inhibited glucose uptake, lactate release and glucose transporter 1 expression in A549/DDP cells compared with those in the control cells. PRDM14 overexpression relieved the inhibitory effects of 3-bromopyruvate, a potent glycolytic inhibitor for glycolysis, on glucose uptake and lactate release in A549 cells compared with those in the control cells. Therefore, the results of the present study suggested that PRDM14 may inhibit the chemosensitivity and promote glycolysis in human LUAD cells.

\section{Introduction}

Lung cancer is a leading cause of death from cancer worldwide with an annual death rate of $\sim 1,000,000$ individuals and a low 5-year survival rate of $\sim 19 \%$ diagnosed between 2009 and 2015 in the United States, although efforts are increasingly being made to improve these outcomes (1-3). Lung adenocarcinoma (LUAD) is a common type of non-small cell lung cancer (NSCLC) that accounts for $\sim 90 \%$ of all lung cancer cases (2). Unfortunately, no completely effective therapeutic methods for the treatment of lung cancer are currently available.

In clinical practice, platinum-based combined chemotherapy is used as the essential pharmacotherapy for patients with lung cancer who need adjuvant chemotherapy or for patients whose cancer is in an advanced, nonoperative state (4). However, chemotherapy failure and a poor prognosis that impairs the quality of life of patients with lung cancer occur frequently due to acquired chemotherapy resistance (4). Thus, studying the mechanism by which chemoresistance develops is urgently required to identify effective therapeutic targets and strategies.

Glycolysis is a glucose metabolism pathway with a high amount of glucose utilization and a low level of oxygen consumption, which is essential for cancer cells to achieve a high rate of proliferation and to avoid apoptosis (5-7). Glycolysis is involved in cancer progression as well as chemoresistance $(5,6)$. Similarly to solid cancers, LUAD exhibits a metabolic switch toward glycolysis $(8,9)$. Accordingly, the dynamic expression of certain molecules involved in glycolysis, such as glucose transporters (GLUT), sodium/glucose 
cotransporters and hexokinase, has been observed in lung cancer (10-12). In addition, previous studies have reported an association between glycolysis and cancer cell survival and between cisplatin (DDP) sensitivity and poor prognosis of patients with lung cancer, suggesting that glycolysis predicts patient overall survival and chemotherapy failure $(8,13-15)$. However, the mechanism between glycolysis and DDP sensitivity is poorly understood at present.

PR domain zinc finger protein (PRDM) family proteins have been demonstrated to regulate cell differentiation, especially in embryonic stem cells (16-18). Recently, PRDM14, a PR-domain-containing transcriptional regulator, has been reported to be differentially expressed in various types of cancer such as NSCLC and breast cancer and to be involved in cancer cell differentiation as well as cancer stemness, migration and metastasis (19-21). However, studies on whether PRDM14 affects chemoresistance and glycolysis in LUAD cells are limited.

The present study aimed to determine the functions of knockdown or overexpression of PRDM14 in the chemosensitivity and glycolysis of LUAD cells. The results may offer an insightful perspective on the function of PRDM14 in glycolysis-mediated chemoresistance of LUAD.

\section{Materials and methods}

Clinical subjects and samples. A total of 40 patients with LUAD (20 DDP-sensitive and 20 DDP-resistant), 22 female and 18 male, aged between 28 and 67 years (median, 48 years), whose first-line treatment was DDP were recruited at The Seventh People's Hospital (Shanghai, China) between March 2014 and October 2017. Using the proportion of changes in tumor volume after treatment, the patients were grouped into four subgroups: i) Complete response (CR; no tumor); ii) partial response (PR; tumor shrinkage by $>50 \%$ ); iii) stable disease (tumor shrinkage by $<50 \%$ or tumor enlargement by $<25 \%$ ); and iv) progressive disease (PD; tumor enlargement by $>25 \%$ ). Patients in the CR and PR groups were defined as DDP-sensitive, and those in the SD and PD groups were defined as DDP-resistant. A total of 20 normal adjacent non-tumor lung tissues, which were resected within at $\geq 5 \mathrm{~cm}$ of the tumor margin when the patients (10 DDP-sensitive and 10 DDP-resistant) underwent surgery, were used as the control samples. All tissues were immediately frozen with liquid nitrogen until further experiments. In addition, a total of 12 primary LUAD cell samples were isolated from 12 patients with LUAD recruited at The Seventh People's Hospital (Shanghai, China) between March 2014 and October 2017, 7 female and 5 male, aged between 35 and 61 years (median, 42 years), as previously described (22) and were classified on the basis of median PRDM14 mRNA expression levels relative to GAPDH mRNA expression levels determined by reverse transcription quantitative (RT-q)PCR into low and high PRDM14 level groups (median value, 3.16). Cases that received preoperative radiochemotherapy before surgical resection were excluded. Written informed consent was obtained from all patients. The study was approved by the Ethics Committee of The Seventh People's Hospital (approval no. 2014-002).

Cell lines and culture. The cell lines used in the present study were obtained from JRDUN Biotechnology
(Shanghai) Co., Ltd., including two human LUAD cell lines, A549 and A549/DDP, and a human bronchial epidermal $16 \mathrm{HBE}$ cell line, which was used as the control. The cells were cultured in the basal culture medium comprising RPMI-1640 medium (HyClone; Cytiva) mixed with $10 \%$ fetal bovine serum (Gibco; Thermo Fisher Scientific, Inc.) at $37^{\circ} \mathrm{C}$ with $5 \% \mathrm{CO}_{2}$ in a cell incubator with a humidified atmosphere.

Vector construction of PRDM14 overexpression and short hairpin (sh)RNA interference. To overexpress PRDM14, the specific coding sequence was synthesized and cloned into pLVX-Puro plasmids (Clontech Laboratories, Inc.). To knock down PRDM14, the specific shRNA sequences targeting PRDM14 (shRNA-1 5'-CCTCATGCAGACGGT GTTT-3'; shRNA-2, 5'-GGATATTCCTGTGAGCCTT-3'; and shRNA-3, 5'-GCATACTCCGCACACACAT-3') or scramble shRNA (5'-CATTCCGCAGTGGTGCATT-3') were cloned into linearized pLKO.1 plasmids (Addgene, Inc.). To produce transducer plasmids, the recombinant plasmids $(1,000 \mathrm{ng})$ were transfected along with the packaging plasmids psPAX2 (100 ng) and pMD2G (900 ng; Addgene, Inc.) and amplified in 293T cells (ATCC; ACS-4500) plated in a 9-well plate $\left(1 \times 10^{5}\right.$ cells/well) for $6 \mathrm{~h}$ at $37^{\circ} \mathrm{C}$. The transfection procedures used Lipofectamine ${ }^{\circledR} 2000$ (Invitrogen; Thermo Fisher Scientific, Inc.) according to the manufacturer's instructions. Subsequently, $48 \mathrm{~h}$ after transfection, the recombinant lentivirus in the cell supernatant was collected by centrifugation at 5,000 $\mathrm{xg}$ for $5 \mathrm{~min}$ and the purification and titration of recombinant lentivirus was performed as previously described (23). A549 or A549/DDP cells were plated in a 6 -well plate $\left(5 \times 10^{5}\right.$ cells/well) and infected with the recombinant lentivirus-transducing units at an MOI of 20 in the presence of $8 \mu \mathrm{g} / \mathrm{ml}$ polybrene (Sigma-Aldrich; Merck KGaA) for $24 \mathrm{~h}$ at $37^{\circ} \mathrm{C}$. Stable cells were selected by puromycin ( $3 \mu \mathrm{g} / \mathrm{ml}$; Thermo Fisher Scientific) for four more days. pLKO.1-scrambled shRNA (shNC) and blank pLVX-Puro (vector) were used as negative controls.

Cell Counting Kit-8 (CCK-8) cell viability assay. The primary LUAD, A549 and A549/DDP cells were plated in a 96-well plate $\left(3 \times 10^{3}\right.$ cells/well $)$ and cultured overnight at $37^{\circ} \mathrm{C}$. The following day, primary LUAD cells were exposed to $10 \mu \mathrm{M}$ DDP (Selleck Chemicals) for $48 \mathrm{~h}$. A549 cells were transduced with the PRDM14 overexpression vector (oePRDM14) and treated with 10 or $20 \mu \mathrm{M}$ DDP or $20 \mu \mathrm{M}$ antiglycolytic agent 3-Bromopyruvate (3-BrPA; MedKoo Biosciences, Inc.) for $48 \mathrm{~h}$ at $37^{\circ} \mathrm{C}$. A549/DDP cells were transfected with shPRDM14 and treated with 10 or $20 \mu \mathrm{M} \mathrm{DDP}$ for $48 \mathrm{~h}$ at $37^{\circ} \mathrm{C}$. Following treatment, $10 \mu$ l CCK-8 solution (Signalway Antibody LLC) was added to each well for $1 \mathrm{~h}$ according to the manufacturer's instructions. The optical density (OD) value of each well was read on a microplate reader at $450 \mathrm{~nm}$ to calculate relative cell viability inhibition rate as follows: Viability inhibition rate $=\left(\mathrm{OD}_{\text {vehicle }+ \text { vector }}-\mathrm{OD}_{\mathrm{DDP}+\text { vector }}\right) / \mathrm{OD}_{\text {vehicle }+ \text { vector }} \mathrm{x} 100 \%$.

Apoptosis analysis following DDP treatment by flow cytometry. A549 and A549/DDP cells were cultured in a 6 -well plate $\left(3 \times 10^{5}\right.$ cells/well) until they reached $50 \%$ confluency. Subsequently, the cells were subjected to transduction or drug treatment. Cells were collected at $48 \mathrm{~h}$ post-treatment. 
The staining procedure included a 15 -min incubation at $4{ }^{\circ} \mathrm{C}$ with $5 \mu \mathrm{l}$ annexin-V-FITC, followed by a 15 -min incubation with $5 \mu$ l propidium iodide (PI; all from Beyotime Institute of Biotechnology). An Accuri ${ }^{\mathrm{TM}}$ C6 flow cytometer (BD Biosciences) using CellQuest Pro software, version 3.3 (Becton, Dickinson and Company) was used to examine early [(annexin-V-FITC)+/PI-] and late [(annexin-V-FITC)+/PI+] apoptosis.

Measurement of glucose uptake. A549 and A549/DDP cells $\left(5 \times 10^{5}\right.$ cells/well $)$ were cultured in a 6 -well plate, kept in continuous cell culture for $24 \mathrm{~h}$ at $37^{\circ} \mathrm{C}$ and subjected to transduction or drug treatment. At $48 \mathrm{~h}$ post-treatment, low-glucose DMEM (Hyclone; Cytiva) was added to each well. Following 3-h incubation, the Krebs-Ringer Bicarbonate Buffer (Guangzhou Weibo Biological Technology Co Ltd.) supplemented with $2 \%$ bovine serum albumin (Beijing Solarbio Science \& Technology Co., Ltd.) was used to replace the culture medium and wash the cells. Subsequently, glucose-free DMEM supplemented with $100 \mu \mathrm{M}$ 2-[N-(7-nitrobenz-2-oxa-1,3-diazol-4-yl) amino]-2-deoxyglucose (2-NBDG; Cayman Chemical Company) was used to culture cells for an additional $45 \mathrm{~min}$, and cell glucose uptake analysis was performed using a flow cytometer.

Measurement of lactate. A549 and A549/DDP cells were seeded in a 6 -well plate $\left(5 \times 10^{5}\right.$ cells/well), cultured for $24 \mathrm{~h}$ and subjected to transfection or drug treatment for $48 \mathrm{~h}$. The medium was collected for the analysis of lactate concentration using a Lactic Acid Assay kit (Nanjing Jiancheng Bioengineering Institute) according to the manufacturer's instructions.

$R T q-P C R$. Total RNA was isolated from the tissue homogenate and cell lines using TRIzol ${ }^{\circledR}$ reagent (Thermo Fisher Scientific, Inc.). The RNA was subjected to reverse transcription into cDNA by using a PrimeScript ${ }^{\mathrm{TM}}$ kit (Takara Biotechnology Co., Ltd.). The cDNA synthesis conditions were $37^{\circ} \mathrm{C}$ for $60 \mathrm{~min}$, followed by $85^{\circ} \mathrm{C}$ for $5 \mathrm{~min}$ and $4^{\circ} \mathrm{C}$ for $5 \mathrm{~min}$. The PCR amplification of cDNA product was performed using the SYBR ${ }^{\circledR}$ Green PCR Master Mix (Applied Biosystems; Thermo Fisher Scientific, Inc.) according to the manufacturer's instructions. The primer sequences were as follows: PRDM14 forward, 5'-GACTCACGCCTGTAATCC-3' and reverse, 5'-GTCTCCTGTGCTCAAACC-3'; and GAPDH forward, 5'-AATCCCATCACCATCTTC-3' and reverse, 5'-AGGCTG TTGTCATACTTC-3'. The fold-changes of the PRDM14 mRNA levels were calculated by the $2^{-\Delta \Delta C q}$ method (24). GAPDH mRNA was used as the internal control. The PCR cycling conditions were $95^{\circ} \mathrm{C}$ for $10 \mathrm{~min}$ followed by 40 cycles at $95^{\circ} \mathrm{C}$ for $15 \mathrm{sec}$ and $60^{\circ} \mathrm{C}$ for $45 \mathrm{sec}$ followed by a final extension step of $95^{\circ} \mathrm{C}$ for $15 \mathrm{sec}, 60^{\circ} \mathrm{C}$ for $1 \mathrm{~min}, 95^{\circ} \mathrm{C}$ for $15 \mathrm{sec}$ and $60^{\circ} \mathrm{C}$ for $15 \mathrm{sec}$.

Western blotting. Total protein was extracted from the LUAD tissue homogenate or cell lines using RIPA lysis buffer supplemented with a mixture of protease inhibitors (Sigma-Aldrich; Merck KGaA). Total protein concentration in each tissue sample was measured using a Lowry protein assay kit (Bio-Rad Laboratories, Inc.). Equivalent quantities $(25 \mu \mathrm{g})$ of protein was separated by 10 or $15 \%$ SDS-PAGE, transferred to a nitrocellulose membrane (EMD Millipore), blocked in $5 \%$ fat-free milk overnight at $4^{\circ} \mathrm{C}$ and incubated with primary antibodies against PRDM14 (1:500; cat. no. ab187881), $\gamma-\mathrm{H} 2 \mathrm{~A}$ histone family member X ( $\gamma$-H2AX; 1:500; cat. no. ab26350), cleaved caspase-3 (1:500; cat. no. ab2302), pro-caspase-3 (1:500; cat. no. ab32150), cleaved poly(ADP-Ribose) polymerase 1 (PARP1; 1:500; cat. no. ab32064), PARP1 (1:500; ab191217) and GLUT1 (1:1,000; cat. no. ab32551) (all from Abcam) overnight at $4^{\circ} \mathrm{C}$, and then washed three times with Tris-buffered saline with $0.1 \%$ Tween-20 (Amresco, LLC). The membranes were subsequently incubated with anti-rabbit horseradish peroxidase-conjugated immunoglobulin G secondary antibody $(1: 1,000$; cat. no. A0208; Beyotime Institute of Biotechnology; ) for $1 \mathrm{~h}$ at $37^{\circ} \mathrm{C}$. GAPDH $(1: 2,000$.; cat. no. 5174; Cell Signaling Technology, Inc.) served as the internal control. Finally, the specific signals were visualized using an enhanced chemiluminescence system (Bio-Rad Laboratories, Inc.) and quantified by densitometry (Quantity One software, version 4.62; Bio-Rad Laboratories, Inc.).

Xenograft establishment with stable cell lines and DDP treatment. A total of $80 \mathrm{BALB} / \mathrm{c}$ nude mice (male; age, 6-8 weeks; weight, 20-25 g) obtained from Shanghai SLAC Laboratory Animal Co., Ltd. were randomly divided into two groups ( $n=40$ per group), and their flank regions were subcutaneously injected with $5 \times 10^{6}$ A549/DDP cells transfected with shPRDM14 or shNC. The animals were maintained at a constant temperature of $25^{\circ} \mathrm{C}$ with $\sim 50 \%$ humidity under a regular 12:12-h light/dark cycle with food and water available ad libitum.

To examine the xenograft sensitivity to DDP, each group was evenly divided into two further subgroups receiving an intraperitoneal injection of $5 \mathrm{mg} / \mathrm{kg}$ DDP or an equal volume of vehicle once a week for 3 weeks ( $n=20$ per group). The xenograft tumors were measured 12 days after the cells were injected, and the tumor volumes were calculated. Tumor xenografts of mice in each group ( $n=6$ per group) were dissected 33 days after cell injection for visual evaluation of morphology and were weighed. Tumor volume was calculated twice a week using the following equation: Volume $=\left(\right.$ length $\mathrm{x}$ width $\left.{ }^{2}\right) / 2$. The remaining mice ( $n=14$ per group) were used to analyze survival rates for 90 days after cell injection.

The animals were sacrificed by an intraperitoneal injection of sodium pentobarbital (30 mg/kg; Vetoquinol UK, Ltd.) followed by cervical dislocation on days 33 and 90 or when they reached certain humane endpoints (tumor diameter $>2 \mathrm{~cm}$ or $20 \%$ body weight loss). Any procedure performed on mice followed the guidelines of the Ethics Committee of Institutional Animal Care and Use Committee of The Seventh People's Hospital (approval no. 2019-078).

Statistical analysis. Data are presented as the mean \pm standard deviation. Statistical analyses were performed by GraphPad Prism 8.0.2 (GraphPad Software, Inc.). Comparisons between two groups were performed using unpaired Student's t-test and among multiple groups using one- or two-way ANOVA followed by Bonferroni or Tukey's multiple comparisons test when the data were compared among multiple groups or with a single control group, respectively. Mouse survival 
A
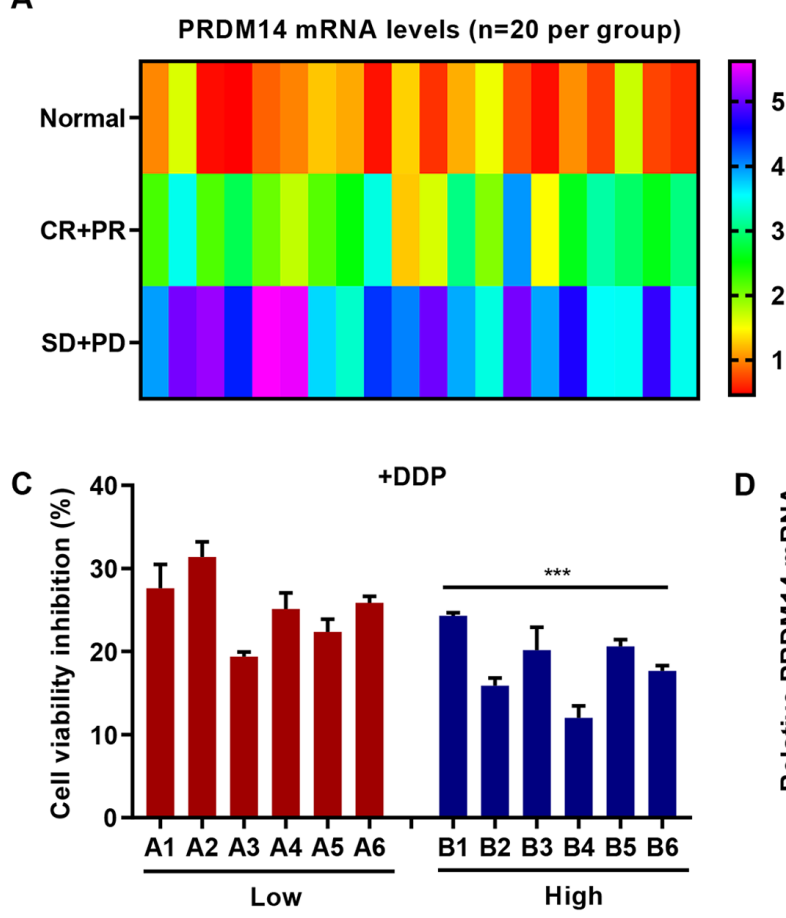

B

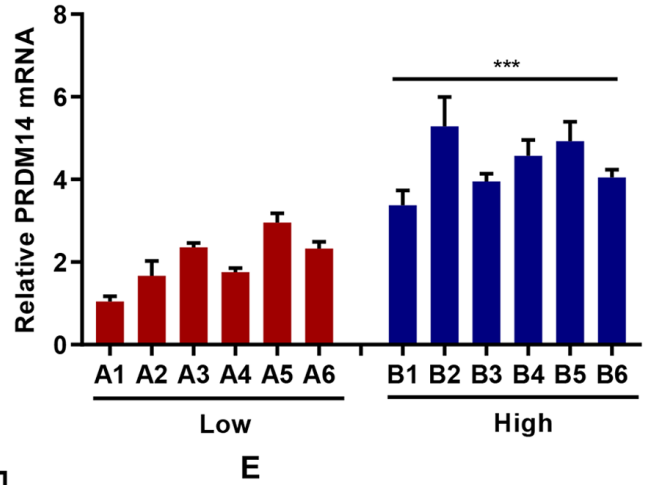

D

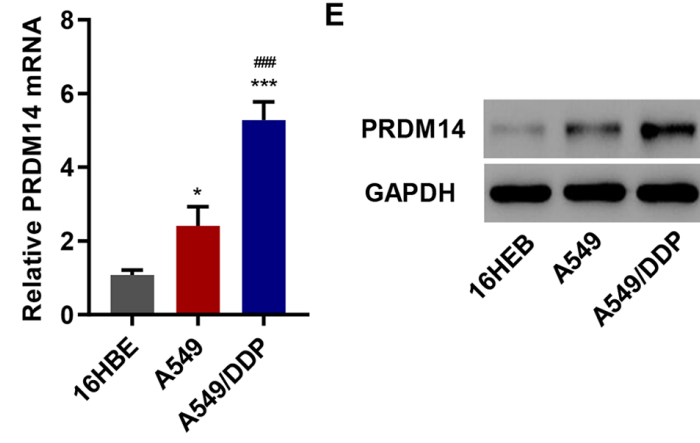

Figure 1. PRDM14 is highly expressed in patients with DDP-resistant LUAD and the A549/DDP cell line. (A) PRDM14 expression in primary LUAD tissues collected from patients with LUAD (20 DDP-sensitive, 20 DDP-resistant) and in normal lung tissues ( $\mathrm{n}=20$ ) was determined by reverse transcription quantitative-PCR. (B) Primary LUAD cells collected from patients with LUAD ( $\mathrm{n}=12$ ) were classified into two groups (low and high PRDM14 expression). (C) Cell viability at $48 \mathrm{~h}$ following treatment with $10 \mu \mathrm{M}$ DDP. ${ }^{* * *} \mathrm{P}<0.001 \mathrm{vs.} \mathrm{low} \mathrm{group.} \mathrm{(D} \mathrm{and} \mathrm{E)} \mathrm{PRDM14} \mathrm{expression} \mathrm{in} \mathrm{LUAD} \mathrm{cell} \mathrm{lines} \mathrm{A549} \mathrm{and} \mathrm{A549/DDP} \mathrm{and}$ human bronchial epithelial $16 \mathrm{HEB}$ cells. ${ }^{*} \mathrm{P}<0.05$ and ${ }^{* * * *} \mathrm{P}<0.001$ vs. $16 \mathrm{HEB}$ cells; ${ }^{\# \# \# / 1} \mathrm{P}<0.001$ vs. A549 cells. DDP, cisplatin; LUAD, lung adenocarcinoma; PRDM14, PR domain zinc finger protein 14; CR, complete response; PR, partial response; SD, stable disease; PD, progressive disease.

was analyzed by the Kaplan-Meier method and log-rank test. Bonferroni correction was used to adjust P-values for multiple measures. Cell experiments were performed in triplicate. $\mathrm{P}<0.05$ was considered to indicate a statistically significant difference.

\section{Results}

PRDM14 expression levels are upregulated in patients with DDP-resistant LUAD and A549/DDP cells. RT-qPCR results demonstrated that patients in the DDP-resistant group expressed higher levels of PRDM14 compared with patients in the DDP-sensitive group (Fig. 1A). To further determine the association between PRDM14 level and the sensitivity of LUAD cells to DDP, 12 primary LUAD cell lines, collected from 12 patients with LUAD, were classified into low and high PRDM14 expression groups (Fig. 1B) and treated with DDP. Following treatment, low cell viability inhibition was observed in the high PRDM14 expression group compared with that in the low PRDM14 group (Fig. 1C). Additionally, in the A549/DDP cells, the expression levels of PRDM14 were higher level compared with those in the progenitor A549 cells (Fig. 1D and E). These data suggested a potential relationship between the chemosensitivity of LUAD cells to DDP and PRDM14.

PRDM14 regulates $A 549$ and A549/DDP cell sensitivity to $D D P$ in vitro. To determine the regulatory effects of PRDM14 on the chemosensitivity of LUAD cells to DDP, PRDM14 was overexpressed in A549 cells (Fig. 2A), and cell viability and the percentage of apoptotic cells sensitive to DDP treatment were measured. In the vector-transfected control cells, 10 and $20 \mu \mathrm{M}$ DDP inhibited cell viability and promoted the apoptotic rate, whereas in cells overexpressing PRDM14, these changes were significantly attenuated (Fig. 2B and C). In A549 cells, the cell viability inhibition rate in response to DDP in the oePRDM14 group $(10 \mu \mathrm{M}, 10.65 \pm 1.99 \% ; 20 \mu \mathrm{M}$, $19.93 \pm 0.26 \%)$ was significantly lower compared with that in the vector group $(10 \mu \mathrm{M}, 15.67 \pm 0.99 \% ; 20 \mu \mathrm{M}, 27.97 \pm 0.83 \%$; Fig. 2B). By contrast, in A549/DDP cells, PRDM14 silencing by shRNA targeting PRDM14 (Fig. 2D) enhanced the decrease in cell viability and the increase in the percentage of apoptotic cells in response to 10 and $20 \mu \mathrm{M}$ DDP (Fig. 2E and F). In A549/DDP cells, the cell viability inhibition rate response to DDP in the shPRDM14 group $(10 \mu \mathrm{M}, 24.16 \pm 2.39 \% ; 20 \mu \mathrm{M}$, $41.67 \pm 3.39 \%)$ was significantly higher compared with that in the shNC group $(10 \mu \mathrm{M}, 17.98 \pm 1.49 \% ; 20 \mu \mathrm{M}, 29.3 \pm 1.38$; Fig. 2E).

Chemosensitivity is associated with not only the activation of apoptosis, but also with DNA damage (25). Therefore, the expression of $\gamma-\mathrm{H} 2 \mathrm{AX}$, a marker of DNA damage, was also determined. In the vector-transfected cells, the two doses of DDP promoted an increase in the expression levels of $\gamma$-H2AX compared with those in the vehicle group, whereas in cells overexpressing PRDM14, this increase was significantly attenuated (Fig. 2G). By contrast, in A549/DDP cells, PRDM14 silencing enhanced the increase in the $\gamma-\mathrm{H} 2 \mathrm{AX}$ expression levels in response to the two doses of DDP compared with 

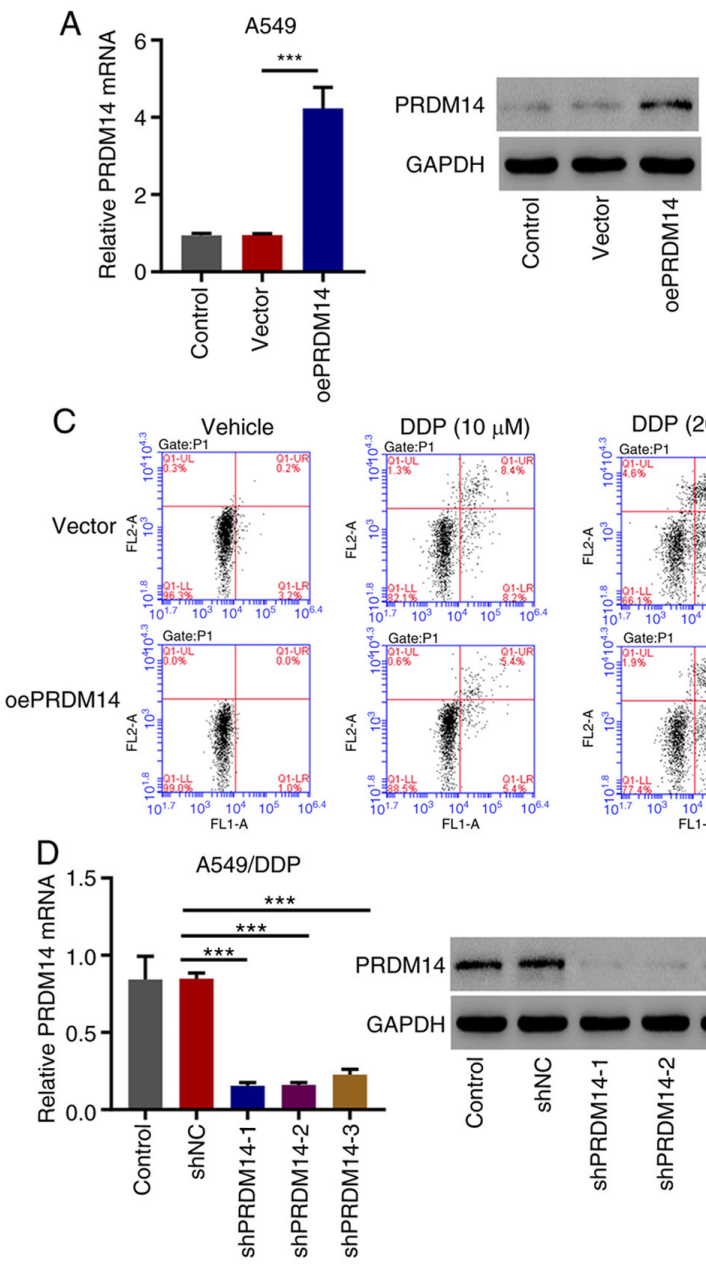

B
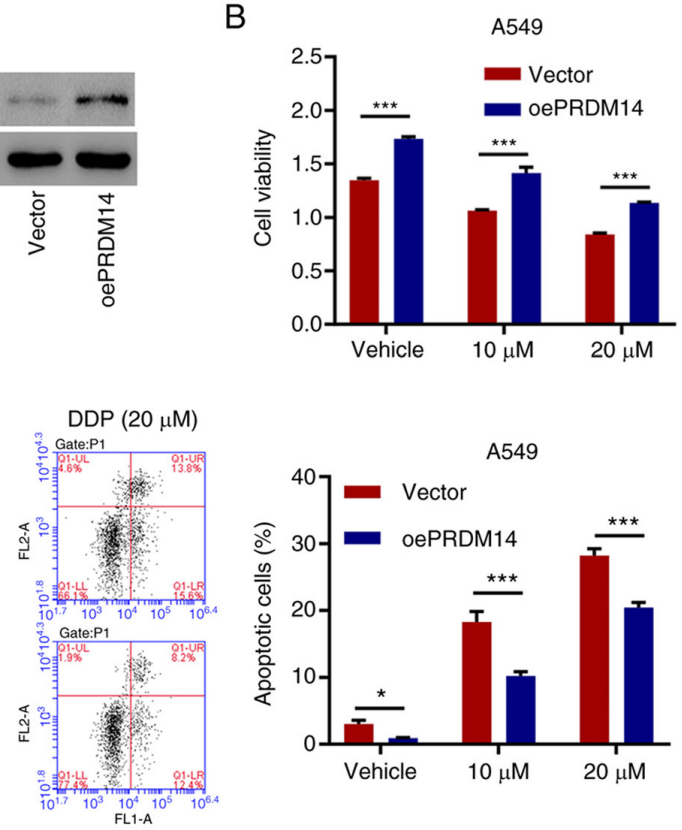

$\mathrm{E}$

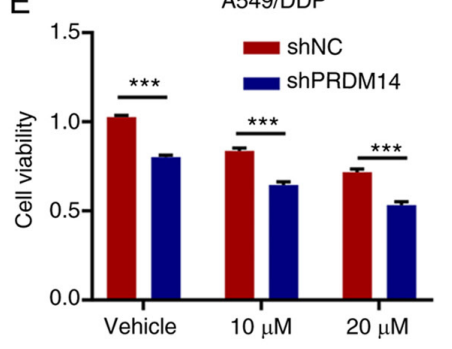

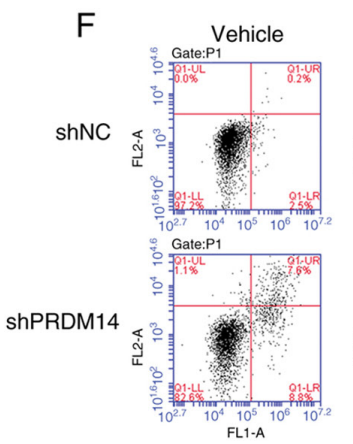
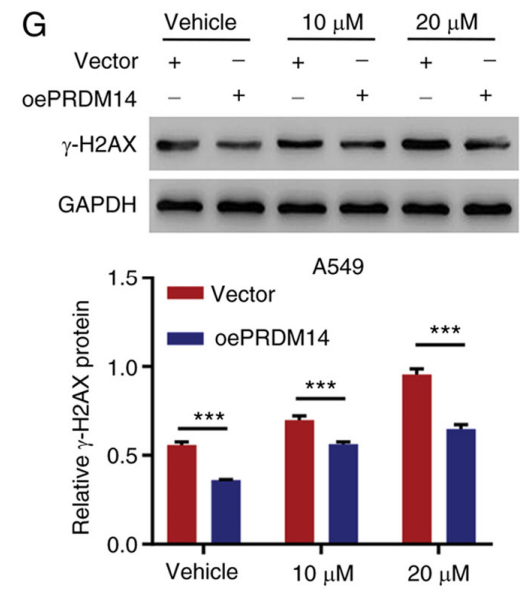
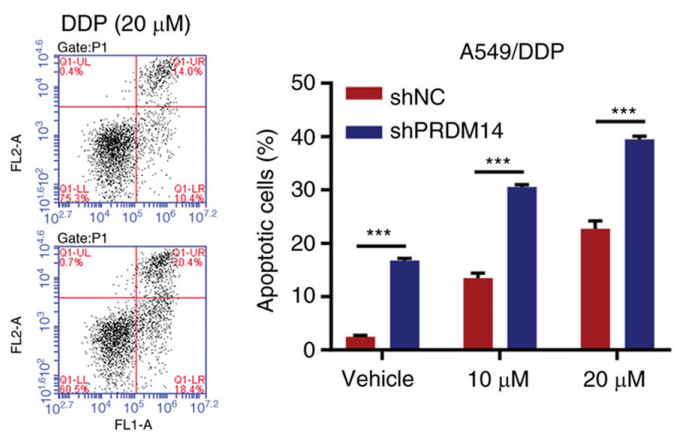

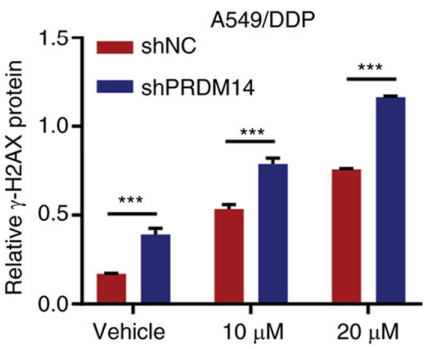

Figure 2. PRDM14 regulates the chemosensitivity of A549 and A549/DDP cells to DDP in vitro. (A and D) Expression of PRDM14 in (A) A549 and (D) A549/DDP cells transfected with the oePRDM14, shPRDM14 or the corresponding controls. (B and E) Cell viability, (C and F) apoptosis and (G) $\gamma$-H2AX expression of A549 and A549/DDP cells transfected with the indicated plasmids treated with 10 and $20 \mu \mathrm{M}$ DDP or vehicle. "P $<0.05$ and ${ }^{* * * *} \mathrm{P}<0.001$. DDP, cisplatin; oe, overexpression; sh, short hairpin; NC, negative control; $\gamma$-H2AX, $\gamma$-H2A histone family member X; PRDM14, PR domain zinc finger protein 14. 
A

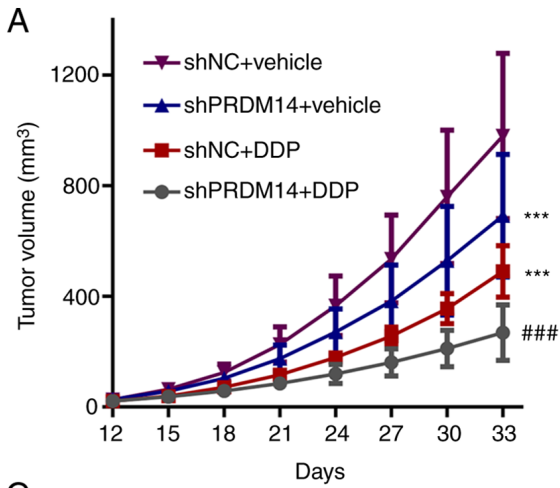

C

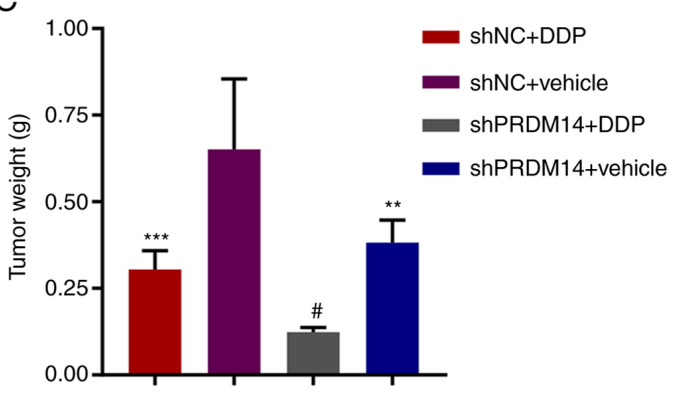

B

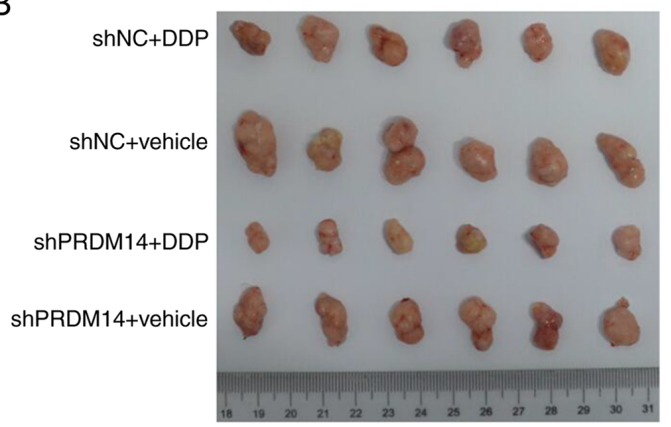

D

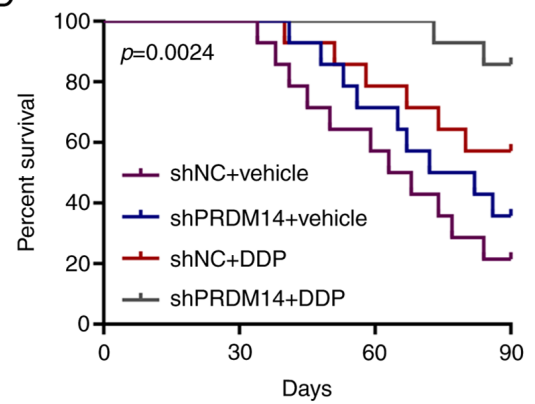

Figure 3. Knockdown of PRDM14 suppresses DDP-resistant tumor growth in vivo. (A) Tumor volume in each group at the indicated time after cell inoculation $(n=6)$. (B and C) Xenografts were collected and weighed on day 33 after tumor inoculation $(n=6)$. (D) Mouse survival rate $(n=14) .{ }^{* *} P<0.01$ and ${ }^{* * *} \mathrm{P}<0.001$ vs. shNC + vehicle on day $33 ;{ }^{\#} \mathrm{P}<0.05$ and ${ }^{\# \# \#} \mathrm{P}<0.001$ vs. shNC + DDP on day 33. DDP, cisplatin; sh, short hairpin; NC, negative control; PRDM14, PR domain zinc finger protein 14 .

that in the vehicle group (Fig. 2G). These results suggested that PRDM14 overexpression inhibited the DDP sensitivity of A549 cells, whereas PRDM14 silencing enhanced that of A549/DDP cells.

Knockdown of PRDM14 restrains DDP-resistant tumor growth in vivo. A model using mice bearing a DDP-resistant tumor with A549/DDP cells transfected with shPRDM14 or shNC was established and treated with DDP treatment to evaluate the role of PRDM14 knockdown in the tumor sensitivity to DDP. As presented in Fig. 3A-C, PRDM14 knockdown alone or DDP alone suppressed tumor growth, whereas PRDM14 knockdown combined with DDP treatment resulted in the lowest tumor volume and weight at 33 days among all groups, suggesting that PRDM14 knockdown enhanced the chemosensitivity of A549/DDP cells to DDP. In addition, PRDM14 knockdown resulted in the highest survival rate of tumor-bearing mice receiving DDP treatment among all groups (Fig. 3D).

PRDM14 silencing inhibits glucose uptake and lactate release in A549/DDP cells. To assess the involvement of PRDM14 in glycolysis in LUAD cells, PRDM14 was silenced by shPRDM14 transfection. The results of glycolysis analysis demonstrated that, compared with the negative control shRNA, PRDM14 silencing reduced the glucose uptake (Fig. 4A and B) and lactate production (Fig. 4C). Additionally, the levels of GLUT1, a protein essential for glucose transport during glycolysis (26), were also repressed by PRDM14 silencing compared with those in the shNC group (Fig. 4D). These results suggested that PRDM14 silencing inhibited the glycolysis of A549/DDP cells.
PRDM14 alleviates the antiglycolytic and pro-apoptotic effects of 3-BrPA in A549 cells. To determine the role of PRDM14 in cell viability and glycolysis, A549 cells were treated with the PRDM14 overexpression vector and 3-BrPA, which is a potent glycolysis inhibitor and a specific anticancer agent for lung tumorigenesis $(27,28)$. The antiglycolytic agent 3-BrPA alone exhibited a significant inhibitory effect on cell viability, glucose uptake, lactate release and the expression levels of pro-caspase-3, PARP1 and GLUT1, but increased the expression levels of cleaved caspase-3 and cleaved PARP1 compared with the untreated group. PRDM14 overexpression alone exerted the opposite effects; PRDM14 overexpression combined with 3-BrPA reversed the inhibitory effects of 3-BrPA in A549 cells (Fig. 4E-I), suggesting that PRDM14 overexpression alleviated the antiglycolytic and pro-apoptotic effects of 3-BrPA.

\section{Discussion}

DDP-based combined chemotherapy is an essential strategy for lung cancer treatment, which fails to improve patient survival rates due to chemotherapy resistance (4). Identifying the key molecules or signaling pathways that control chemoresistance is an urgent research target. The results of the present study demonstrated that PRDM14, a protein highly expressed in DDP-resistant patients and in LUAD cells, negatively controlled the sensitivity of LUAD cells to DDP by promoting glycolysis, suggesting that PRDM14 may be a target for chemotherapy in patients with LUAD.

PRDM14 exhibits a low expression level in adjacent-normal tissues in adults, but is upregulated in various types of cancer, whereas aberrant PRDM14 expression 
A
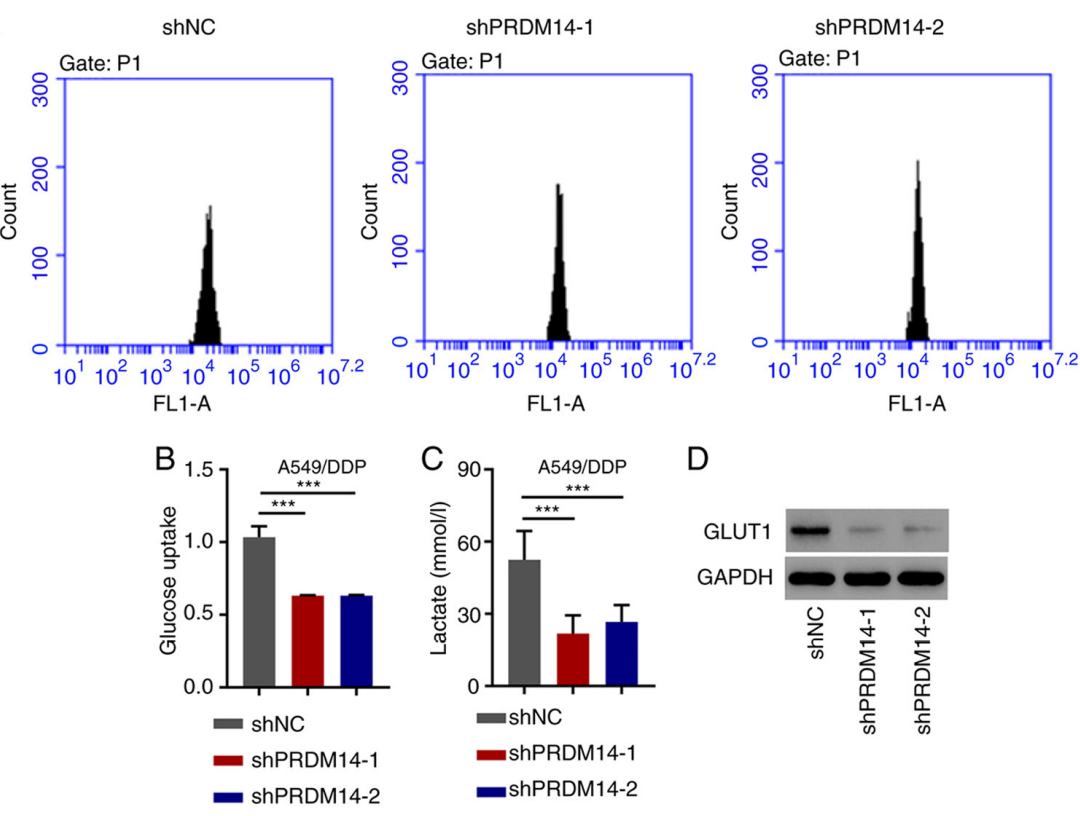

D

GLUT1 GAPDH
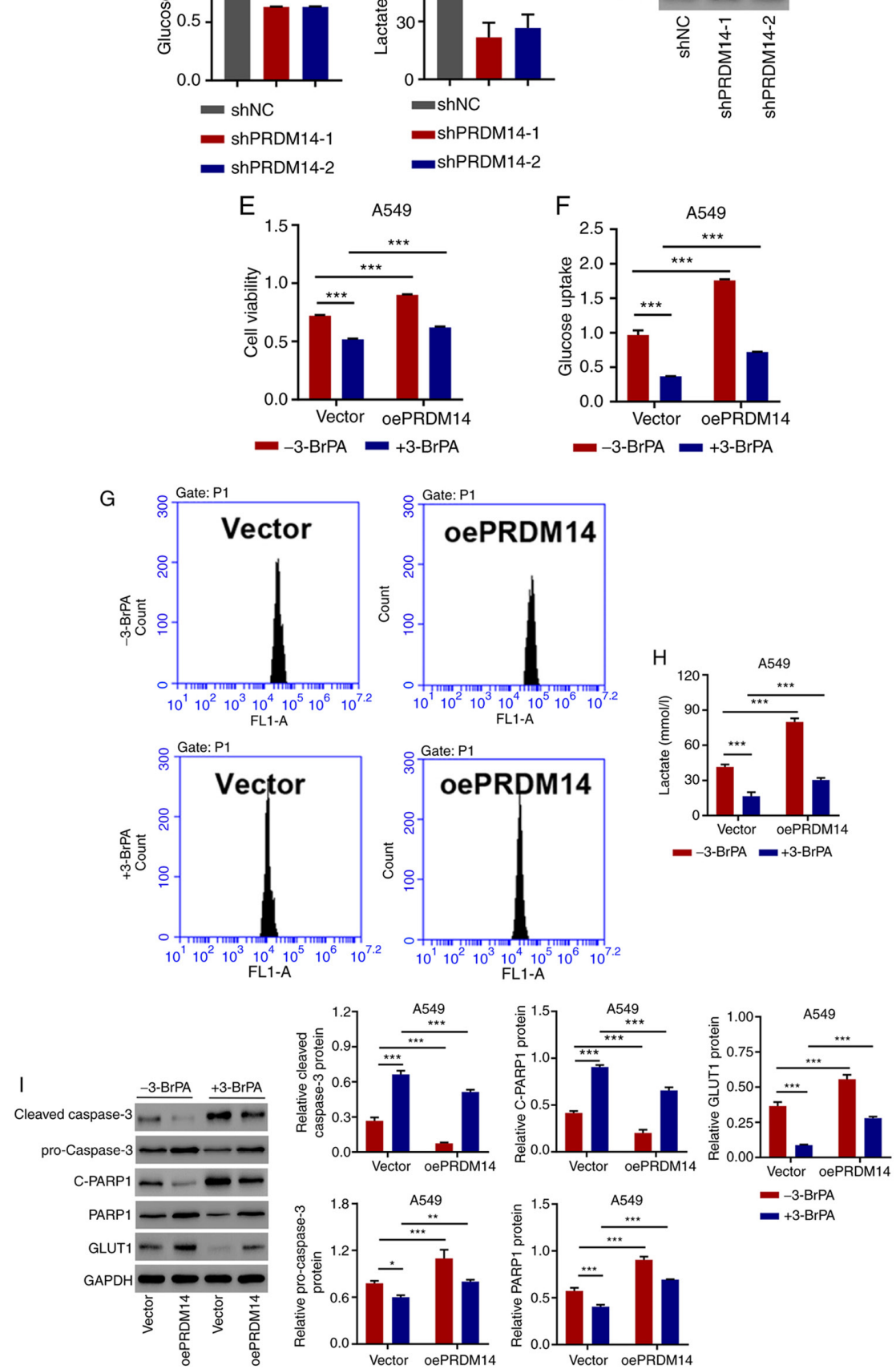

Figure 4. PRDM14 regulates glucose uptake and lactate release in A549/DDP cells and alleviates the effects of 3-BrPA in A549 cells. (A and B) Glucose uptake, (C) lactate release and (D) GLUT1 expression in A549/DDP cells transfected with shPRDM14 or shNC. (E) Cell viability, (F and G) glucose uptake and (H) lactate release, and (I) C-caspase-3, pro-caspase-3, C-PARP1, PARP1 and GLUT1 expression levels in A549 cells transduced with oePRDM14 or blank vector in the absence or presence of the antiglycolytic agent 3-BrPA. ${ }^{*} \mathrm{P}<0.05,{ }^{* *} \mathrm{P}<0.01$ and ${ }^{* * *} \mathrm{P}<0.001$. DDP, cisplatin; oe, overexpression; sh, short hairpin; NC, negative control; GLUT, glucose transporter; C-, cleaved; PARP1, poly(ADP-ribose) polymerase 1; 3-BrPA, 3-bromopyruvate; PRDM14, PR domain zinc finger protein 14. 
controls the cancer cell phenotype and acts as a driver of oncogenic processes, such as in colorectal (29), pancreatic (30) and breast (21) cancer. Similarly, the results of the present study demonstrated that PRDM14 was upregulated in patients with DDP-resistant LUAD and A549/DDP cells, and enhanced the resistance of LUAD cells to DDP. Since limited studies have reported the function of PRDM14 in the chemoresistance of LUAD cells, the present study focused on PRDM14 and explored its underlying mechanism in this process.

A previous study has demonstrated that PRDM14 regulates the cell migration and invasion in human NSCLC (20). The metabolic priorities of cancer cells differ from those of normal cells, thus providing a new therapeutic window; the Warburg effect, also termed glycolysis, is characterized by high glucose uptake and lactate release, and is considered to be a hallmark of prostate cancer (31). This metabolic adaptation benefits cancer cells in surviving through hypoxic conditions, which are common in tumors, and supports their anabolic requirements (32). Elaborating the central role of PRDM14 in the glycolysis of DDP-resistant lung cancer cells is an important outcome of the present study. Aerobic glycolysis is actively promoted by cancer cells to sustain the metabolic requirements for tumor progression as well as in cells escaping damage from chemotherapeutic agents $(33,34)$, which has been demonstrated in the chemoresistance of lung cancer $(9,35)$. Notably, the results of the present study demonstrated that PRDM14 silencing inhibited glucose uptake and lactate release in A549/DDP cells and that PRDM14 overexpression restored the glycolysis of A549 cells under the inhibition by antiglycolytic agent 3-BrPA, indicating that PRDM14 may positively promote the chemoresistance of LUAD cells by increasing glycolysis.

Despite an increasing number of studies focusing on the role of PRDM14 in tumor progression and chemoresistance, the understanding of the targets of PRDM14 is still limited. Previous studies have attempted to determine the interaction between PRDM14 and gene associated with cell proliferation, apoptosis and glycolysis, which are crucial cancer cell processes during chemoresistance (36-38). PRDM14 may affect the proliferation of $293 \mathrm{~T}$ cells by inducing cell cycle arrest at G1/S phase (36), target apoptosis regulators phorbol-12-myristate-13-acetate-induced protein 1 [PMAIP1, also known as NOXA) and $\mathrm{Bcl}-2$ binding component 3 (BBC3, also known as PUMA)] in the apoptosis evasion of HPV-positive cancer cells (37), and interact with heat shock protein $90 \alpha$ and glucose-regulated protein 78 , two molecules associated with chemotherapy resistance (38).

The present study analyzed the regulatory effects of PRDM14 on the expression of caspase-3, which is a pro-apoptotic gene (39), and GLUT1, which is a protein mediating glucose transport, metabolism and chemoresistance of NSCLC cells (26). The activation of caspase-3 and PARP1 and the downregulation of GLUT1 induced by 3-BrPA was reversed by PRDM14 overexpression in A549 cells, suggesting that caspase-3 and GLUT1 may be downstream targets for PRDM14. However, whether PRDM14 regulates caspase-3 and GLUT1 via its transcriptional activity directly or other indirectly mechanisms require further validation in future studies.
There were several limitations to the present study that need to be considered when interpreting these results. First, although this cohort quite accurately reflected the normal span of patients in a clinic, the sample size was quite small. Second, clinical parameter information necessary to assess a correlation between PRDM14 expression and LUAD progression needs to be further validated. Finally, the pathways should be clarified in further investigation, especially those involved in the apoptosis, glycolysis and chemoresistance-related signals.

In conclusion, the results of the present study suggested the key role of PRDM14 in the chemoresistance of LUAD cells and demonstrated the regulatory effects of PRDM14 on apoptosis and glycolysis in LUAD cells resistant to DDP. The results of the present study provide new insights and support the application of PRDM14 as a chemotherapy target for LUAD.

\section{Acknowledgements}

Not applicable.

\section{Funding}

This work was funded by Natural Science Foundation of China (grant no. 81904044), Talents Training Program of Seventh People's Hospital of Shanghai University of TCM (grant no. XX2017-06), Shanghai Health Commission Youth Project (grant no. 20174Y0044), Key Disciplines Construction Project of the Municipal Health Commission, Pudong New Area (grant no. PWZxk2017-06) and the Budget Project of Shanghai University of Traditional Chinese Medicine (grant no. 2019LK045).

\section{Availability of data and materials}

The datasets used and/or analyzed during the current study are available from the corresponding author on reasonable request.

\section{Authors' contributions}

SH, XM and DY performed the experiments. NZ and GW acquired, analyzed and interpreted the data. MW and WX designed the study and drafted the manuscript. All authors read and approved the final manuscript.

\section{Ethics approval and consent to participate}

The present study was approved by the Ethics Committee of The Seventh People's Hospital (approval no. 2014-002), and written informed consent was obtained from each patient. Procedures performed on mice followed the guidelines of the Ethics Committee of Institutional Animal Care and Use Committee of The Seventh People's Hospital (approval no. 2019-078).

\section{Patient consent for publication}

Not applicable. 


\section{Competing interests}

The authors declare that they have no competing interests.

\section{References}

1. Pan X, Chen Y, Shen Y and Tantai J: Knockdown of TRIM65 inhibits autophagy and cisplatin resistance in A549/DDP cells by regulating miR-138-5p/ATG7. Cell Death Dis 10: 429, 2019.

2. Stankovic B, Bjørhovde HAK, Skarshaug R, Aamodt H, Frafjord A, Müller E, Hammarström C, Beraki K, Bækkevold ES Woldbæk PR, et al: Immune cell composition in human non-small cell lung cancer. Front Immunol 9: 3101, 2018.

3. Siegel RL, Miller KD and Jemal A: Cancer statistics, 2020. CA Cancer J Clin 70: 7-30, 2020.

4. Rossi A and Di Maio M: Platinum-based chemotherapy in advanced non-small-cell lung cancer: Optimal number of treatment cycles. Expert Rev Anticancer Ther 16: 653-660, 2016.

5. Li FL, Liu JP, Bao RX, Yan GQ, Feng X, Xu YP, Sun YP, Yan W, Ling ZQ, Xiong Y, et al: Acetylation accumulates PFKFB3 in cytoplasm to promote glycolysis and protects cells from cisplatin-induced apoptosis. Nat Commun 9: 508, 2018.

6. Bose S and Le A: Glucose metabolism in cancer. Adv Exp Med Biol 1063: 3-12, 2018.

7. Vander Heiden MG, Cantley LC and Thompson CB: Understanding the Warburg effect: The metabolic requirements of cell proliferation. Science 324: 1029-1033, 2009.

8. Kim JH, Nam B, Choi YJ, Kim SY, Lee JE, Sung KJ, Kim WS, Choi CM, Chang EJ, Koh JS, et al: Enhanced glycolysis supports cell survival in EGFR-Mutant lung adenocarcinoma by inhibiting autophagy-mediated EGFR degradation. Cancer Res 78: 4482-4496, 2018.

9. Zhang W, Bouchard G, Yu A, Shafiq M, Jamali M, Shrager JB, Ayers K, Bakr S, Gentles AJ, Diehn M, et al: GFPT2-expressing cancer-associated fibroblasts mediate metabolic reprogramming in human lung adenocarcinoma. Cancer Res 78: 3445-3457, 2018.

10. Higashi K, Yamagishi T, Ueda Y, Ishigaki Y, Shimasaki M, Nakamura Y, Oguchi M, Takegami T, Sagawa M and Tonami H: Correlation of HIF-1 $\alpha /$ HIF-2 $\alpha$ expression with FDG uptake in lung adenocarcinoma. Ann Nucl Med 30: 708-715, 2016.

11. Huber SM, Misovic M, Mayer C, Rodemann HP and Dittmann K: EGFR-mediated stimulation of sodium/glucose cotransport promotes survival of irradiated human A549 lung adenocarcinoma cells. Radiother Oncol 103: 373-379, 2012.

12. Ciribilli Y, Singh P, Inga A and Borlak J: c-Myc targeted regulators of cell metabolism in a transgenic mouse model of papillary lung adenocarcinoma. Oncotarget 7: 65514-65539, 2016.

13. Gong T, Cui L, Wang H, Wang H and Han N: Knockdown of KLF5 suppresses hypoxia-induced resistance to cisplatin in NSCLC cells by regulating HIF-1 $\alpha$-dependent glycolysis through inactivation of the PI3K/Akt/mTOR pathway. J Transl Med 16: $164,2018$.

14. Liu J, Lu F, Gong Y, Zhao C, Pan Q, Ballantyne S, Zhao X, Tian S and Chen H: High expression of synthesis of cytochrome c oxidase 2 and TP53-induced glycolysis and apoptosis regulator can predict poor prognosis in human lung adenocarcinoma. Hum Pathol 77: 54-62, 2018.

15. Zhang L, Zhang Z and Yu Z: Identification of a novel glycolysis-related gene signature for predicting metastasis and survival in patients with lung adenocarcinoma. J Transl Med 17: 423, 2019.

16. Tan SX, Hu RC, Xia Q, Tan YL, Liu JJ, Gan GX and Wang LL: The methylation profiles of PRDM promoters in non-small cell lung cancer. Onco Targets Ther 11: 2991-3002, 2018.

17. Fog CK, Galli GG and Lund AH: PRDM proteins: Important players in differentiation and disease. Bioessays 34: 50-60, 2012.

18. Hohenauer T and Moore AW: The prdm family: Expanding roles in stem cells and development. Development 139: 2267-2282, 2012.

19. Taniguchi $\mathrm{H}$ and Imai K: PRDM14, a zinc finger protein, regulates cancer stemness. Methods Mol Biol 1867: 3-13, 2018.

20. Bi HX, Shi HB, Zhang T and Cui G: PRDM14 promotes the migration of human non-small cell lung cancer through extracellular matrix degradation in vitro. Chin Med J (Engl) 128 373-377, 2015.

21. Taniguchi H and Imai K: Silencing PRDM14 via oligonucleotide therapeutics suppresses tumorigenicity and metastasis of breast cancer. Methods Mol Biol 1974: 233-243, 2019.
22. Tuthill MH, Montinaro A, Zinngrebe J, Prieske K, Draber P, Prieske S, Newsom-Davis T, von Karstedt S, Graves J and Walczak H: TRAIL-R2-specific antibodies and recombinant TRAIL can synergise to kill cancer cells. Oncogene 34: 2138-2144, 2015.

23. Xiong S, Zheng Y, Jiang P, Liu R, Liu X and Chu Y: MicroRNA-7 inhibits the growth of human non-small cell lung cancer A549 cells through targeting BCL-2. Int J Biol Sci 7: 805-814, 2011.

24. Livak KJ and Schmittgen TD: Analysis of relative gene expression data using real-time quantitative PCR and the 2(-Delta Delta C(T)) method. Methods 25: 402-408, 2001.

25. Sakurai Y, Ichinoe M, Yoshida K, Nakazato Y, Saito S, Satoh M, Nakada N, Sanoyama I, Umezawa A, Numata Y, et al: Inactivation of REV7 enhances chemosensitivity and overcomes acquired chemoresistance in testicular germ cell tumors. Cancer Lett 489: 100-110, 2020.

26. Suzuki S, Okada M, Takeda H, Kuramoto K, Sanomachi T, Togashi K, Seino S, Yamamoto M, Yoshioka T and Kitanaka C: Involvement of GLUT1-mediated glucose transport and metabolism in gefitinib resistance of non-small-cell lung cancer cells. Oncotarget 9: 32667-32679, 2018.

27. Fan T, Sun G, Sun X, Zhao L, Zhong R and Peng Y: Tumor energy metabolism and potential of 3-bromopyruvate as an inhibitor of aerobic glycolysis: Implications in tumor treatment. Cancers (Basel) 11:317, 2019.

28. Zhang Q, Pan J, North PE, Yang S, Lubet RA, Wang Y and You M: Aerosolized 3-bromopyruvate inhibits lung tumorigenesis without causing liver toxicity. Cancer Prev Res (Phila) 5: 717-725, 2012.

29. Igarashi $\mathrm{H}$, Taniguchi $\mathrm{H}$, Nosho $\mathrm{K}$, Ishigami $\mathrm{K}$, Koide $\mathrm{H}$, Mitsuhashi K, Okita K, Takemasa I, Imai K and Nakase H: PRDM14 promotes malignant phenotype and correlates with poor prognosis in colorectal cancer. Clin Transl Oncol 22: 1126-1137, 2020

30. Moriya C, Taniguchi H, Miyata K, Nishiyama N, Kataoka K and Imai K: Inhibition of PRDM14 expression in pancreatic cancer suppresses cancer stem-like properties and liver metastasis in mice. Carcinogenesis 38: 638-648, 2017.

31. Liang T, Ye X, Yan D, Deng C, Li Z and Tian B: FAM46B promotes apoptosis and inhibits glycolysis of prostate cancer through inhibition of the MYC-LDHA axis. Onco Targets Ther 13: 8771-8782, 2020.

32. Heydarzadeh S, Moshtaghie AA, Daneshpoor M and Hedayati M: Regulators of glucose uptake in thyroid cancer cell lines. Cell Commun Signal 18: 83, 2020.

33. Chakraborty PK, Mustafi SB, Xiong X, Dwivedi SKD, Nesin V, Saha S, Zhang M, Dhanasekaran D, Jayaraman M, Mannel R, et al: MICU1 drives glycolysis and chemoresistance in ovarian cancer. Nat Commun 8: 14634, 2017.

34. Ganapathy-Kanniappan S and Geschwind JF: Tumor glycolysis as a target for cancer therapy: Progress and prospects. Mol Cancer 12: 152, 2013.

35. Kikuchi R, Iwai Y, Tsuji T, Watanabe Y, Koyama N, Yamaguchi K, Nakamura $\mathrm{H}$ and Aoshiba K: Hypercapnic tumor microenvironment confers chemoresistance to lung cancer cells by reprogramming mitochondrial metabolism in vitro. Free Radic Biol Med 134: 200-214, 2019.

36. Lu Y, Wan Z, Zhang X, Zhong X, Rui L and Li Z: PRDM14 inhibits $293 \mathrm{~T}$ cell proliferation by influencing the G1/S phase transition. Gene 595: 180-186, 2016.

37. Snellenberg S, Cillessen SA, Van Criekinge W, Bosch L, Meijer CJLM, Snijders PJF and Steenbergen RDM: Methylation-mediated repression of PRDM14 contributes to apoptosis evasion in HPV-positive cancers. Carcinogenesis 35: 2611-2618, 2014.

38. Moriya C, Taniguchi H, Nagatoishi S, Igarashi H, Tsumoto K and Imai K: PRDM14 directly interacts with heat shock proteins HSP90 $\alpha$ and glucose-regulated protein 78. Cancer Sci 109: 373-383, 2018

39. Feng Y, Hu J, Xie D, Qin J, Zhong Y, Li X, Xiao W, Wu J, Tao D, Zhang M, et al: Subcellular localization of caspase-3 activation correlates with changes in apoptotic morphology in MOLT-4 leukemia cells exposed to X-ray irradiation. Int J Oncol 27: 699-704, 2005.

This work is licensed under a Creative Commons Attribution-NonCommercial-NoDerivatives 4.0 International (CC BY-NC-ND 4.0) License. 